\title{
Resonance control of mid-infrared metamaterials using arrays of split- ring resonator pairs
}

\author{
Weisheng Yue ${ }^{1}$, Zhihong Wang ${ }^{2}$, John Whittaker ${ }^{1}$, Fredrik Schedin ${ }^{3}$, Zhipeng $\mathrm{Wu}^{4}$ and \\ Jiaguang $\operatorname{Han}^{5}$ \\ ${ }^{1}$ National Graphene Institute, University of Manchester, Oxford Road, Manchester M13 \\ 9PL, United Kingdom \\ ${ }^{2}$ Advanced Nanofabrication Core Lab, King Abdullah University of Science and \\ Technology, Thuwal 23955-6900, Saudi Arabia \\ ${ }^{3}$ Centre for Mesoscience and Nanotechnology, School of Computer Science, University of \\ Manchester, Manchester, M13 9PL, United Kingdom \\ ${ }^{4}$ School of Electrical and Electronic Engineering, University of Manchester, Sackville \\ Street, Manchester M13 9PL, United Kingdom \\ ${ }^{5}$ Centre for Terahertz Waves and College of Precision Instrument and Optoelectronics \\ Engineering, Tianjin University, Tianjin 300072, China \\ E-mail: weisheng.yue@manchester.ac.uk
}

\begin{abstract}
We present our design, fabrication and characterization of resonance controllable metamaterials operating in mid-infrared wavelengths. The metamaterials are composed of pairs of back-to-back or face-to-face U-shape split-ring resonators (SRRs). Transmission spectra of the metamaterials are measured using Fourier transform infrared spectroscopy
\end{abstract}


(FTIR). The results show that the transmission resonance is dependent on the distance between the two SRRs in each SRR-pair. The dips in the transmission spectrum shift to shorter wavelengths with increasing distance between the two SRRs for both the back-toback and face-to-face SRR-pairs. The position of the resonance dips in the spectrum can hence be controlled by the relative position of the SRRs. This mechanism of resonance control offers a promising way for developing metamaterials with tunability for optical filters and bio/chemical sensing devices in integrated nano-optics.

Keywords: Metamaterials; Nanofabrication; Electron-beam lithography; Mid-infrared

\section{Introduction}

Metamaterials are artificially engineered materials that have exotic properties which are not attainable with naturally occurring materials [1]. Novel properties such as negative refraction [1, 2], superlensing [3], electromagnetic cloaking[4], electromagnetically induced transparency $[5,6]$, and so on, have been demonstrated with metamaterials. Applications areas of metamaterials include biochemical sensors, energy harvesting, communications, imaging and others [7-10].

The achievement of tunability of metamaterials is one of the most important aims of metamaterial research. The effective properties of metamaterials are determined by their composing elements rather than by atoms or molecules as in conventional materials [11]. This offers opportunity to control the optical properties through the control of the composing elements. Metallic nanostructures are widely used to compose metamaterials by generating plasmonic resonances $[2,12,13]$. Among various designs, U-shape split-ring resonators (SRRs) are the most commonly used structure for composing metamaterials [14-16]. An SRR 
can be considered as an $L C$ oscillatory circuit. A single-turn magnetic coil forms an inductance $L$ and the gap between the arms of the SRR forms the capacitance $C[8,17]$. The choice of materials and the dimensions of the structure determines the two parameters $L$ and $C$ and thus determines the resonant frequency of the metamaterials. Therefore, previous efforts on resonance control of metamaterials have focused on controlling the geometry of composing SRRs. Changing the geometry of elemental SRR (for example, gap size or symmetricity) leads to a change in one of the parameters $L$ or $C$ and thus a change in the resonance frequency [18-20]. However, those approaches have their limitations. Firstly, a precise control of geometrical shape of single elements is very difficult to achieve, especially for metamaterials operating at infrared or optical frequencies [21]. Secondly, a change in other parameters like periodicity will result in large change of the area of the metamaterials and thus the chip size. In addition, the change of periodicity influences the light-matter interaction within the nanostructures [22]. Several other approaches, such electric control [23], magnetic control [24] and optical control [25], have been reported to control the resonance frequency of metamaterials. These methods require external electrical or magnetic fields to modulate the optical properties, and an additional experimental setup is required. The above-mentioned problems can be partially overcome by simply integrating multiple single elements. In integrated elemental structures, the resonance can be controlled by electromagnetic interaction between the single structures in the elemental unit instead of the shape variations. This design concept have actually been used to design resonance controllable metamaterials by using integrated SRRs with other structures, for example, nanowire-SRRs hybrid structures and SRR-pairs [26, 27]. In the integrated elements, the electromagnetic fields couple between each SRR and other nanostructures. The coupling 
strength influences the resonance frequency of the metamaterials and thus can be used to manipulate the resonance of the metamaterials. Therefore, resonance-controllable metamaterials can be developed by using the relative position of the structures within a cell structure. This design strategy provides opportunities for developing resonance-controllable metamaterials without precise modification of the geometrical shape of single elements which puts very high requirements on the fabrication process and equipment.

In this work, we propose resonance-controllable metamaterials operating in midinfrared regime by using two identical U-shaped SRRs (SRR-pairs). The control of the resonance is achieved by a modification of relative position of the two SRRs in the pairs. The mid-infrared waves are important for many fields such as chemical/biological sensing, thermal imaging and optical communications [28-30]. The gold SRR-pairs are fabricated using electron-beam (e-beam) lithography in combination with plasma etching. The resonance of the metamaterials is investigated with respect to the relative positions of the two SRRs in the SRR-pairs. Finally, numerical simulations were performed using CST microwave Studio which is based on finite integration technique (FIT), and the simulations agree well with the experimental results.

\section{Fabrication and measurements}

The SRR-pairs were fabricated with e-beam lithography on GaAs substrates. E-beam lithography is an ideal method for fabrication of nanostructures [31]. It has very high resolution and is versatile in fabrication of various nanostructures with precisely controlled geometrical shapes. GaAs was chosen as substrate because it has good transmission 
capability at mid-infrared wavelengths. The fabrication process included e-beam lithography and plasma etching. The cleaned GaAs substrates were coated with an $8 \mathrm{~nm}$ titanium (Ti) layer followed by a $50 \mathrm{~nm}$ gold $(\mathrm{Au})$ layer by magnetron sputtering. The Ti layer worked as a promotion layer to improve adhesion between the Au film and the GaAs surface. Negative tone e-beam resist Ma-N2400 (Microresist Technology Gmbh) was used in the fabrication. This resist has good resistance to reactive ion etching. The resist was spin-coated onto the substrates to a thickness of $200 \mathrm{~nm}$. The e-beam lithography was performed by using a CRESTEC 9500C e-beam writer, which has typical electron energy of $50 \mathrm{keV}$. After the development, the exposed SRR patterns were etched with an Oxford PlasmaLab100 etcher. During the etching step, the resist pattern on the Au film works as mask. The Au layer was milled directly by pure argon ions $\left(\mathrm{Ar}^{+}\right)$with gas flow rate of $30 \mathrm{sccm}$ (standard cubic centimeters per minute). Then gases of argon (30 sccm), $\mathrm{SF}_{6}(10 \mathrm{sccm})$ and $\mathrm{CF}_{4}(20 \mathrm{sccm})$ were introduced to the etching chamber to clean the Ti adhesion layer. Finally, the residual resist was removed with acetone immersion followed by oxygen plasma cleaning. A detailed fabrication process is described elsewhere in our publication [32].

Transmission spectra were taken with a commercial Fourier-transform infrared (FTIR) spectroscopy Nicolet iS10 (Thermo Scientific). The wavelengths of the excitation source are $2.5-25 \mu \mathrm{m}$. The sample is measured in a vertical configuration, in which the excitation IR is normal to the sample surface. The incidence beam was polarized with a continuum zinc selenide $(\mathrm{ZnSe})$ IR polarizer. The transmittance was normalized with respect to the transmittance of the blank GaAs substrate.

\section{Design and numerical modeling}


The geometry of the SRR-pairs is depicted in Figure 1. Figure 1 (a) shows the back-to-back SRR-pair (the relative position shift $S$ is positive) and Figure 1(b) shows the face-to-face SRR-pair ( $\mathrm{S}$ is negative). In the designs, the leg length $\mathrm{L}=570 \mathrm{~nm}$, width of Au wire $\mathrm{W}=85$ $\mathrm{nm}$, lateral width $\mathrm{b}=510 \mathrm{~nm}$ and the thickness of the Au nanowires is $50 \mathrm{~nm}$. The periodicity of the array is $\mathrm{P}=1500 \mathrm{~nm}$ in both $x$ and $y$ directions. The relative position of the two SRRs is variable. The light is incident perpendicularly to the plane of the structure with the electric field in the $x$-direction.

We use commercially available software CST Microwave Studio (version 2014) to simulate the spectral response and electromagnetic fields of the SRR-pairs, as shown in Figure 1. The CST Microwave studio solver is based on finite integration technique (FIT) [33]. In the simulation configuration, the electromagnetic wave is incident normal to the sample plane (in z-direction) (see Figure 1). Periodic boundary conditions are applied in the $x$ and $y$ directions to simulate an infinite array in both directions. Perfectly matched layers (PML) conditions are used along the axis of propagation of the electromagnetic waves, The dispersion properties of the $\mathrm{Au}$ were described as the Drude model with formula, $\varepsilon(\omega)=\varepsilon_{\infty}+\omega_{p}^{2} / \omega(\omega-i \gamma)[34]$, where the plasma frequency $\omega_{p}=1.367 \times 10^{16} \mathrm{~Hz}$ and damping frequency $\gamma=6.478 \times 10^{13} \mathrm{~Hz}[34,35]$. The damping influence of the Ti is considered in the simulation. The dispersion parameters of $\mathrm{Ti}$ in mid-infrared frequencies are obtained from literature [36]. The refractive index of the GaAs substrate is taken as 10.78 for the mid infrared wavelength $(\lambda=8 \mu \mathrm{m})[37]$. 

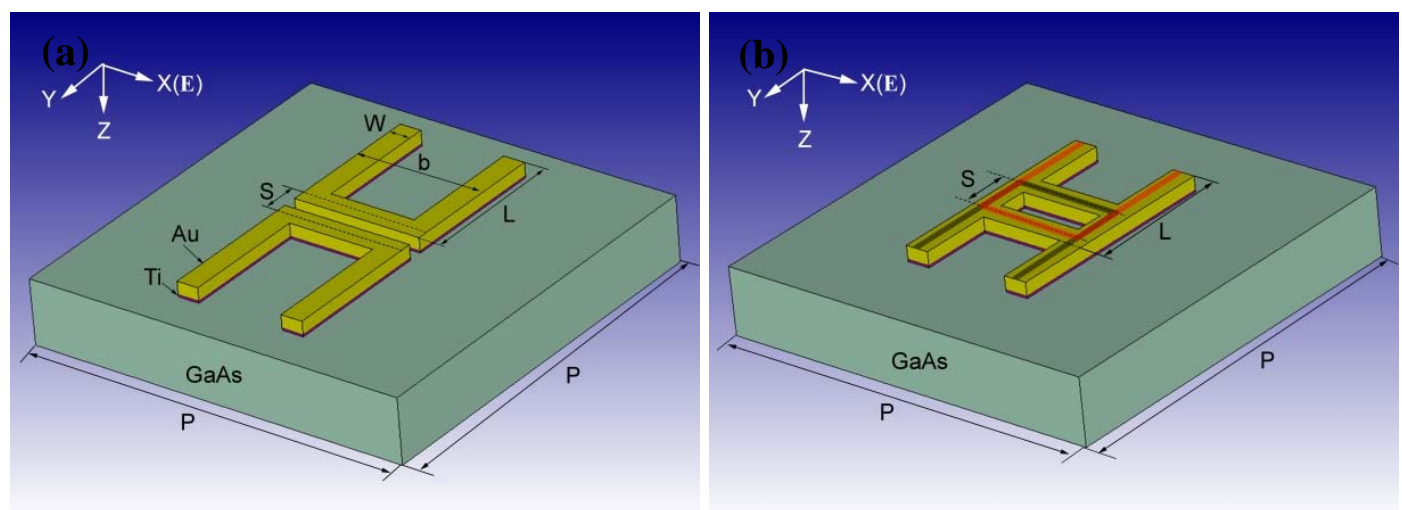

Figure 1. Geometrical schematic of SRR-pairs. (a) back-to-back SRR-pairs, (b) face-toface SRR-pair. The black and red U-shape lines in Figure 1(b) indicate each individual Ushape SRR; $b=510 \mathrm{~nm}, \mathrm{P}=1500 \mathrm{~nm}, \mathrm{~L}=570 \mathrm{~nm}, \mathrm{~W}=85 \mathrm{~nm}$.

\section{Results and Discussion}

Figure 2 presents the SEM images of the fabricated SRR nanostructures. Figure 2 (a-c) show images of the back-to-back SRR-pairs and Figure 2 (d-f) show the images of face-to-face SRR-pairs. The geometrical shapes are well defined due to the high resolution of the e-beam lithography. EDX measurements were performed to confirm that the composition of SRRs is $\mathrm{Au}$ and the EDX spectrum measured on an SRR-pair is shown in Figure 3.
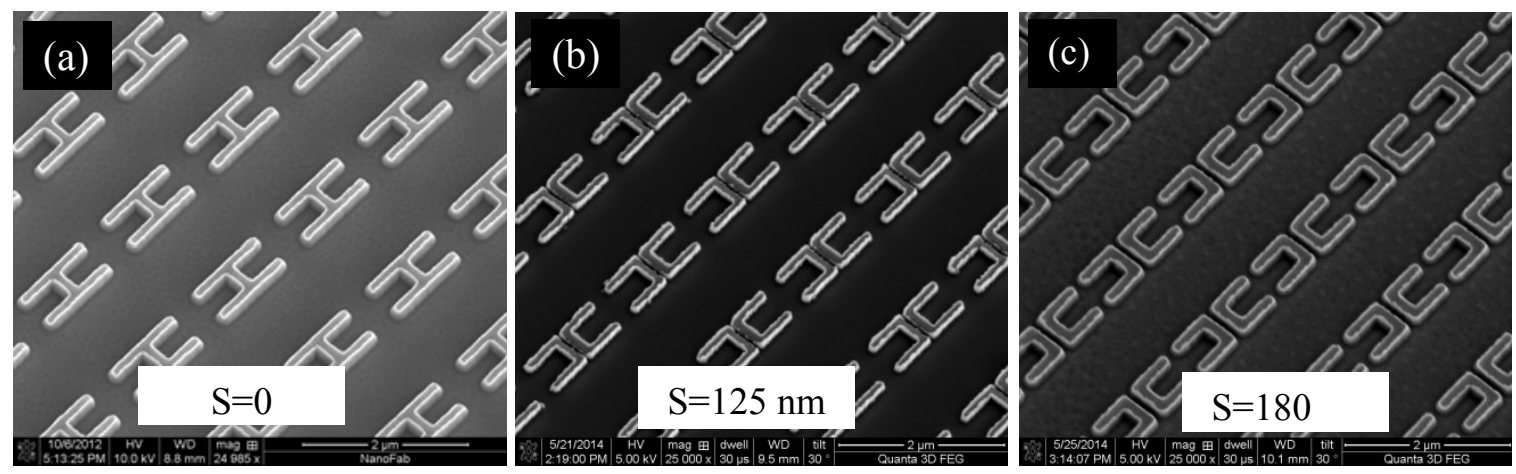

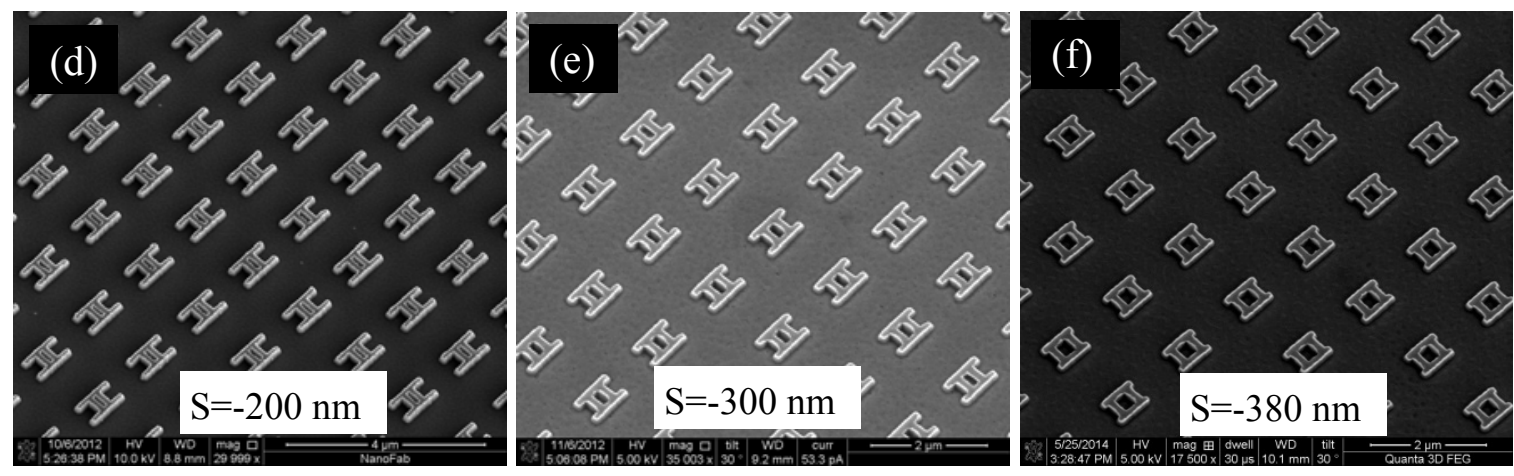

Figure 2. SEM images of fabricated Au SRR-pairs on GaAs substrates.

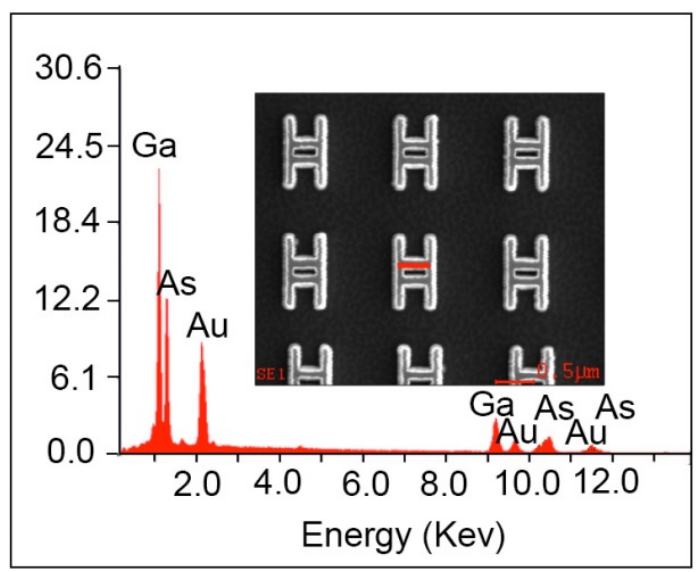

Figure 3. EDX spectrum of an SRR-pair.

Figure 4 (a) shows the measured transmission spectra through the back-to-back SRR-pairs. In these measurements, the polarization (E-vector) of the incident light is along the lateral direction ( $x$-direction) of the SRR-pairs. Sharp resonance dips are observed at 6-7 $\mu \mathrm{m}$ wavelengths, which are primarily determined by the periodicity of the SRR-pairs. The variation in the resonance position is attributed to modification of the electromagnetic interaction as a function of the different relative positions of SRRs in the pairs. The resonance dips blue-shift from 7 and finally down to $5 \mu \mathrm{m}$ when the relative position is increased from 0 , to $125,140,180$, and up to $250 \mathrm{~nm}$. This shift to shorter wavelengths demonstrates a strong 
dependence of the resonance on the relative positon of the two SRRs. Shown in Figure 4(b) are the simulated transmission spectra through the SRR-pairs with the same geometrical parameters. The simulated spectra have similar resonance with dip position and resonance shifting with relative position $S$, revealing a good agreement with the experimental results.
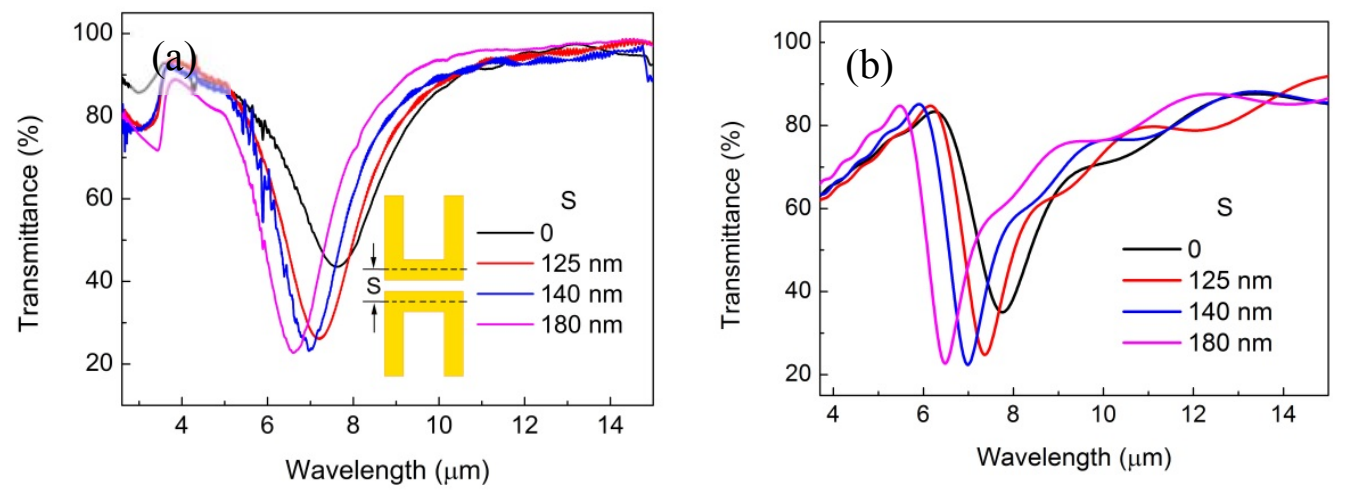

Figure 4. Measured (a) and simulated (b) transmission spectra for the back-to-back SRRpairs.

Figure 5 shows the simulated distribution of the electric field, magnetic field and surface current in a unit cell. The first row in Figure 5 (a1-d1) shows the electric field distribution. The electric field is concentrated around the ends of the four arms and is symmetrical to the vertical center line of the SRR-pairs. The second row in Figure 5 (a2-d2) shows the distribution of the magnetic field. The magnetic field is concentrated at the bottom of each SRR which is generated by the excited circulating current on the Au surface. The third row in Figure $5(a 3-d 3)$ shows the surface current of the surface of the structure. The surface current flow is symmetric with respect to the axis parallel to the bottom of the SRRs and antisymmetric with respect to the axis vertical to the bottom bars. Due to the excitation of surface current on the Au wire, the SRRs work like $L C$ circuits and can be modeled as $L C$ resonators. Figure 6 demonstrate an equivalent $L C$ circuit model of the back-to-back SRR- 
pairs. The self-inductance $L 1$ is caused by the electric current in the Au ring and the capacitance $C 1$ of each SRR is due to the gap region between the SRR arms [14]. Because the two SRRs are proximately placed, coupling between the two SRRs occurs and is described with mutual inductance $L m$ and mutual capacitance $C m$. Then $L$ and $C$ of the system can be written as the sum of two components $L=L 1+L m$ and $C=C 1+C m$. The resonant frequency can be approximated as $\omega \propto 1 /[(L 1+L m)(C 1+C m)]^{1 / 2}$. The $L 1$ and $C 1$ are generally determined by the geometrical shape of the SRR ring. The increase of the relative position $S$ doesn't change the inductance $L 1$ and capacitance $C 1$, as the length of the arms and the split gap remain the same. The mutual inductance $\mathrm{Lm}$ and mutual capacitance $\mathrm{Cm}$ decrease with the increase of the distance $S$, which result in the increase of resonance frequency according to the formula above. This analysis is in agreement with the experimental results that the resonance dips shifts to shorter wavelengths with increase of the relative distance between the two SRRs.

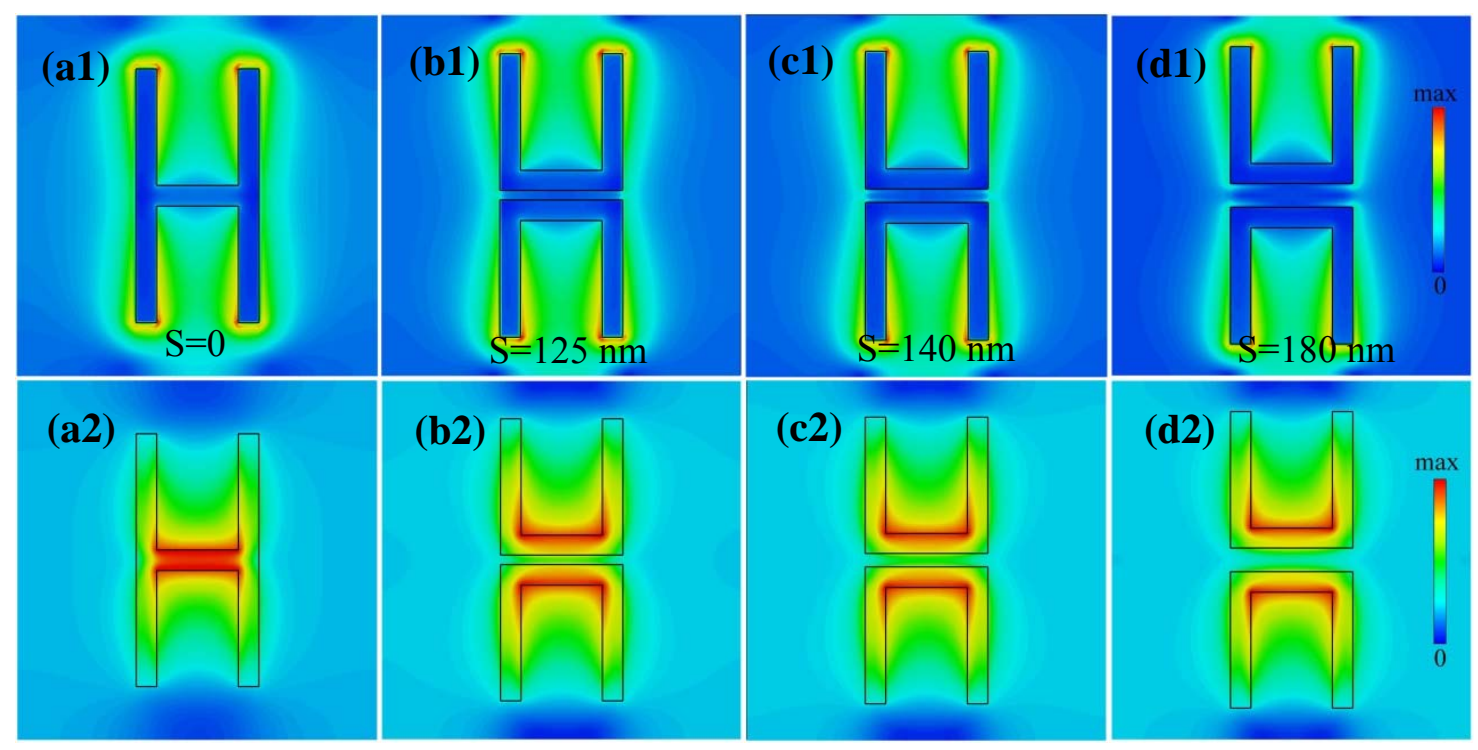




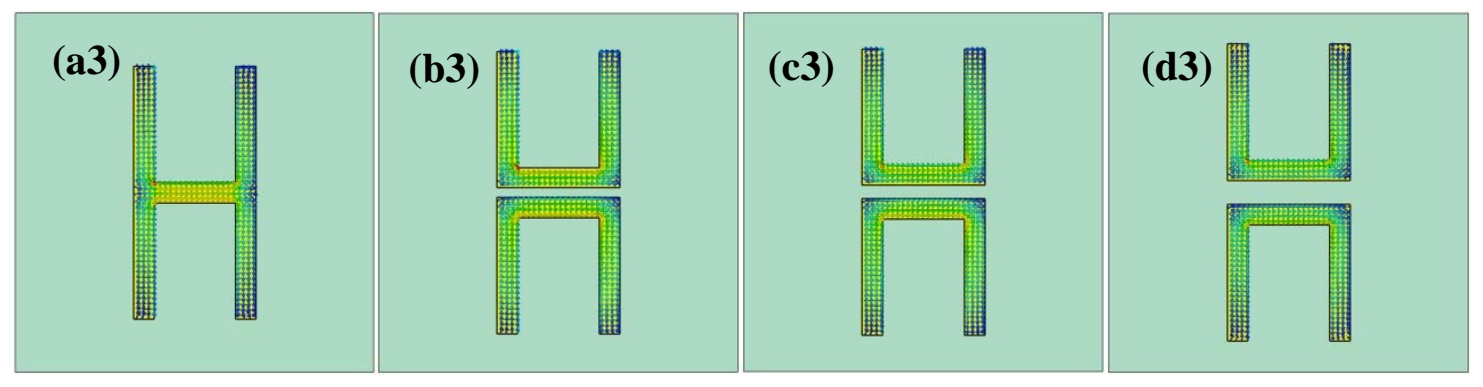

Figure 5. Simulated distributions of electric field (a1-d1), magnetic field (a2-d2) and electric current density (a3-d3) of back-to-back SRR-pairs with different relative position $S$. The field and current distributions are obtained at the resonance wavelength of the metamaterials.

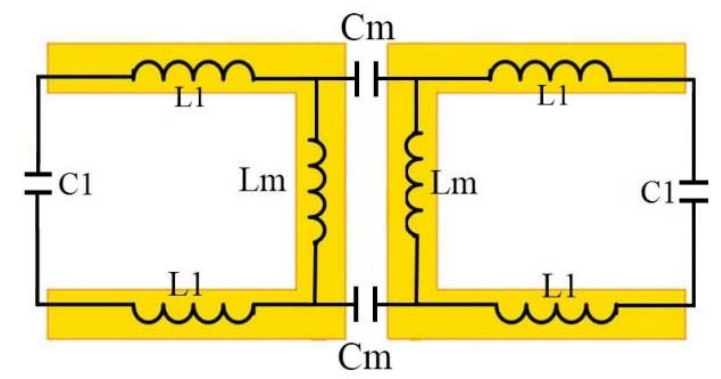

Figure 6. $L C$ circuit model of the back-to-back SRR-pairs.

The resonance properties were also investigated for face-to-face SRR-pairs and the dependence of the resonance on the relative position was also observed here. In the face-toface configuration, the two SRRs are placed oppositely and are merged together. A schematic of the face-to-face SRR-pairs is shown in Figure 1(b). The red and black lines on the surface of Au patterns indicate each individual SRR. Shown in Figure 7(a) and Figure 7(b) are the measured and simulated transmission spectra, respectively. Strong resonance is observed for the face-to-face SRRs. In comparison with the transmission spectra of the back-to-back SRRpairs shown in Figure 4, the transmission spectra of the face-to-face SRR-pairs have two obvious new features: the resonance wavelength is shorter and the resonance band is sharper. This difference can be ascribed to the smaller overall size of the merged face-to-face SRR- 
pair, because scaling down the size of the cell results in a shorter resonant wavelength [38]. Furthermore, in contrast to the back-to-back SRR-pair result, an increase of the relative position $S$, leads to a decrease in the magnitude of the resonance dip. This is because the interaction area decreases with the scaling down of the unit cell. In addition, the increase of $S$ for the face-to-face SRR-pair also modifies the arm length and the size of the closed $\mathrm{Au}$ ring in the middle, and as a consequence changes the electromagnetic interactions. This will be further analyzed with the $L C$ circuit model below. The dependence of the resonance on the relative position of the two SRRs is significant. An increase in the negative direction of the bottom bar distance from the top bar, from 0 , to -200 , to -300 , and to $-380 \mathrm{~nm}$, lead to a shift of the resonance wavelength to shorter wavelengths. The simulated transmission spectra (Figure $7 \mathrm{~b}$ ) are in good agreement with the results observed experimentally.
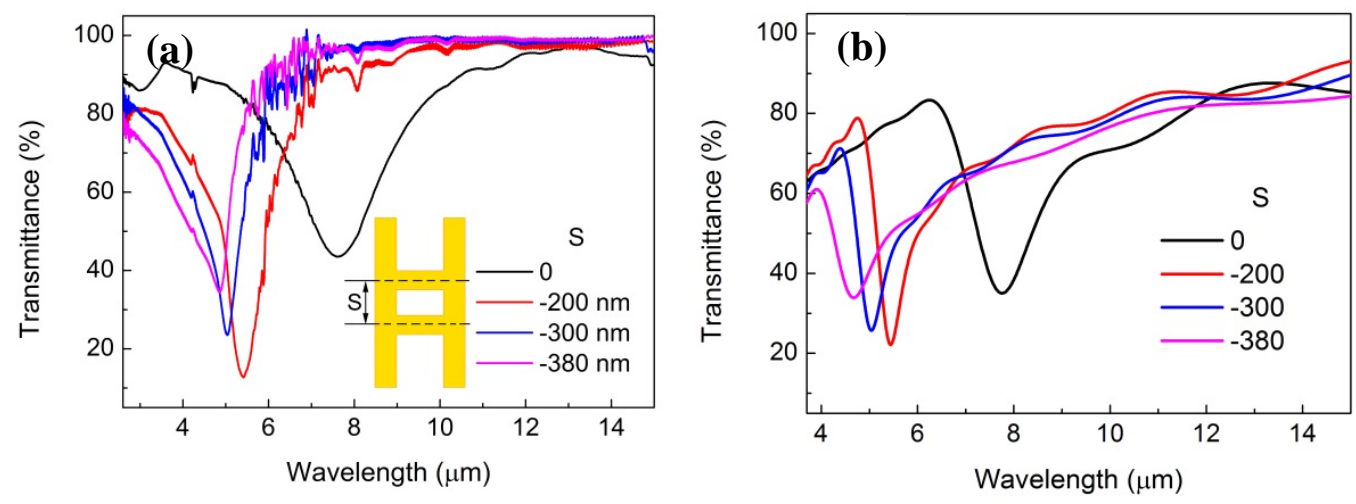

Figure 7. Measured (a) and simulated (b) transmission spectra for the face-to-face SRRpairs.

Figure 8 illustrates the simulated distribution of electric field, magnetic field and surface current density in a unit face-to-face SRR-pairs. High electric field is concentrated around the ends of the four legs (first row), which is similar to that of the back-to-back SRR-pairs shown in Figure 5. Because the two SRRs are merged together, new SRRs on the top and 
bottom as well as a closed Au square ring in the middle are formed. The magnetic field is redistributed on the newly formed SRRs. The magnetic field is confined around the horizontal bars at the bottoms of the SRRs (Figure 8 a2-c2). The third row shows the surface current distributions. The surface current distribution is symmetrical with respect to the horizontal axis in the middle of the SRR-pairs and antisymmetrical with respect to the vertical axis in the middle. This current distribution is similar to that of the back-to-back SRR-pairs. The differences are that the arms of the "U" rings in the face-to-face SRR-pairs are shorter than that of the back-to-back SRR-pairs. In addition, the closed Au loop in the middle distributes part of the current and therefore influences the magnetic field distribution.

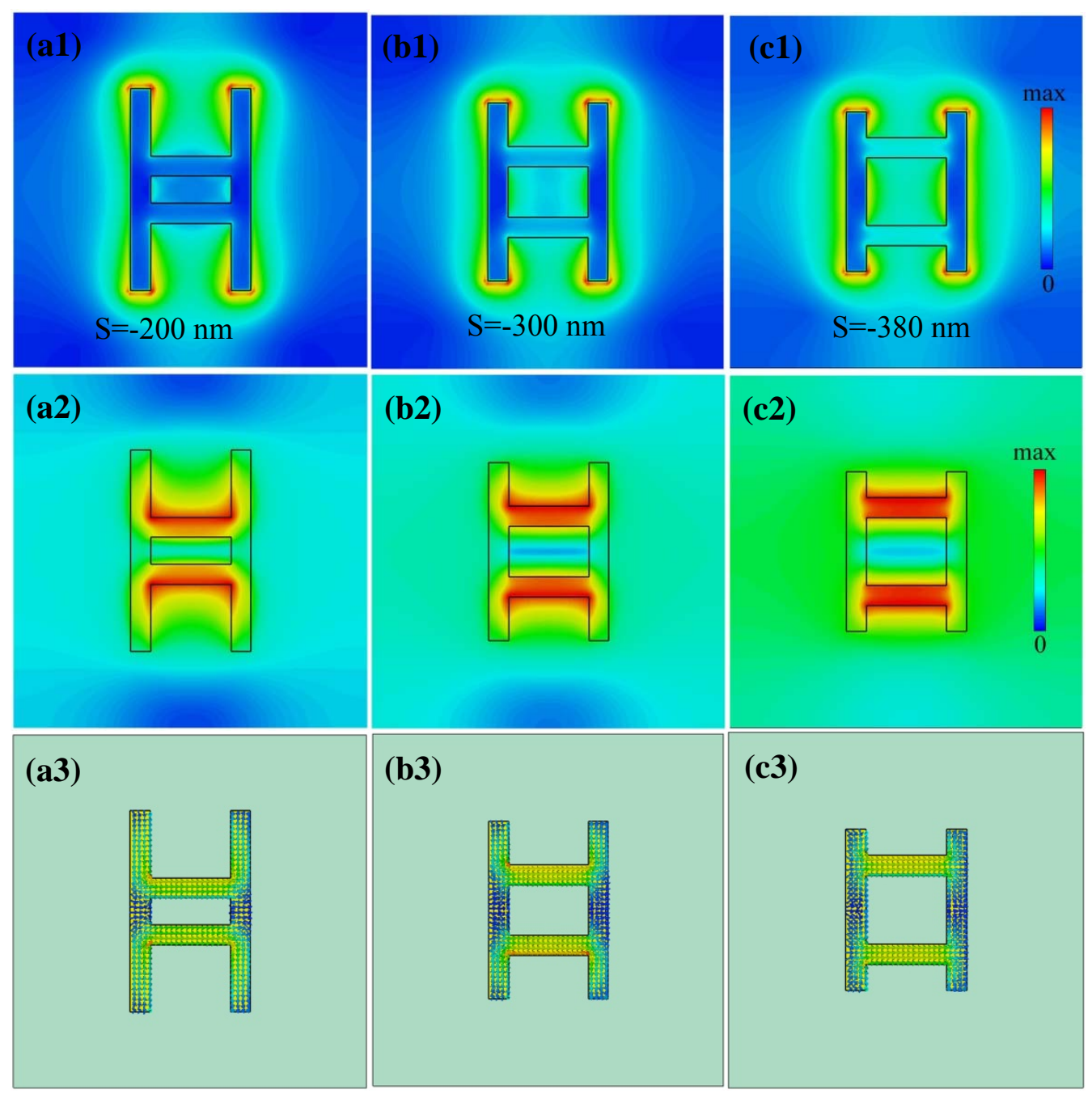


Figure 8. Simulated distributions of the electric field (a1-c1), magnetic field (a2-c2) and surface current (a3-c3) of back-to-back SRR-pairs with different relative position S. The field and current density are obtained at resonance wavelengths.

The dependence of the resonance on the relative position shift $S$ for the face-to-face SRRpairs can also be interpreted with a similar $L C$ circuit model to that used above. The method is described in detail in our previous work [39]. Figure 9 shows the schematic of the LC circuit model. With the increase in relative position, the arm lengths for each SRR are becoming shorter. This change results in the decrease of the self-inductance and capacitance of each SRR and an increase in the mutual capacitance between the two SRRs. The mutual inductance $L m$ remains the same since the length of the horizontal bar does not change. The total effect is that the product of $L C$ decreases and thus the resonance frequency, $\omega \propto 1 / \sqrt{L C}$, increase. This analysis is in agreement with the observed blue-shift with an increase in the relative position.

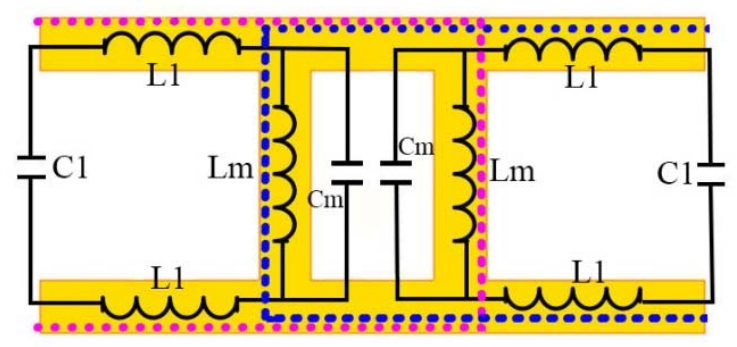

Figure 9. $L C$ circuit model of the face-to-face SRR-pairs. The pink and blue dotted lines indicate each individual SRR. 
Both the back-to-back and face-to-face SRR-pairs have exhibited a strong dependence of their resonances on the relative position of the two U-shape SRRs. Therefore, the resonance of the metamaterials can be controlled the by using the relative position of the two SRRs in the SRR-pairs. Many other methods have been reported to control the resonance wavelength (frequency) in infrared frequencies. For example, by employing the phase transition of vanadium oxide $\left(\mathrm{VO}_{2}\right)$, which is deposited underneath $\mathrm{Au}$ SRRs [26], by electrical modulations of free carrier concentrations in a quantum well layer well beneath the Au split ring resonator array [40] and by tailoring symmetry of the composing elements [20]. To compare the results of our work with these previously reported designs, $Q$-factors were calculated using the formula, $Q=\lambda / \Delta \lambda$, where $\lambda$ is the resonant wavelength and $\Delta \lambda$ is the full-width at half-maximum (FWHM) of the resonance peaks. The $Q$-factors of the resonant band in the transmission spectra in our work are in the range of 4.1-4.3 for the back-to-back SRRs and 4.3-5.4 for the face-to-face SRRs. Table 1 shows comparisons of $Q$-factors with other reported designs. It is seen from the comparison that the $Q$-factors obtained in this work are comparable or higher than that of the other reported designs. This suggests that resonance dips in the transmission spectra in our work are narrower and deeper. These features of resonance are particularly useful in developing various filters and resonance-shifting based molecular sensors. In addition to the narrow bandwidth of the resonance dips, the designs proposed in this work are easier to fabricate, and the experimental setup is simple as there is no requirement to modulate the resonance electrically or optically.

Table 1 Comparisons of the $Q$-factors in this work with other similar metamaterials 


\begin{tabular}{lcccc}
\hline $\begin{array}{c}\text { Resonance-control } \\
\text { method }\end{array}$ & $\begin{array}{c}\text { Typical } \\
\text { elemental } \\
\text { structure }\end{array}$ & $\begin{array}{c}\text { Resonance } \\
\text { wavelengths } \\
(\mu \mathrm{m})\end{array}$ & Q-factors & Reference \\
\hline $\begin{array}{l}\text { shifting of two SRRs } \\
\text { within the SRR-pairs }\end{array}$ & $4.7-7.3$ & $4-5$ & this work \\
$\begin{array}{l}\text { Based on } \mathrm{VO}_{2} \text { phase } \\
\text { transition }\end{array}$ & $2.2-2.5$ & $\sim 2$ & {$[26]$} \\
$\begin{array}{l}\text { Electrical modulation } \\
\text { of } \\
\text { concentration } \\
\begin{array}{l}\text { Tailing symmetry } \\
\text { carrier }\end{array}\end{array}$ & 9.8 & $\sim 3$ & {$[40]$} \\
\end{tabular}

\section{Conclusion}

In conclusion, we have demonstrated geometrically-controlled mid-infrared metamaterial resonances by using SRR-pairs. The resonances are highly dependent on the relative position of the two SRRs within the SRR-pairs. The resonant wavelength shifts towards to shorter wavelengths with an increase in the relative distance between the two SRRs for both the back-to-back and face-to-face SRR-pairs. Numerical simulations are in agreement with the experimental observations. This work provides proof-of-principle experimental evidence, which is useful in designing tunable devices including optical switches, resonance tunable sensors, and filters etc.

\section{References}

[1] Padilla W J, Basov D N and Smith D R 2006 Negative refractive index metamaterials Mater. Today 9 28-35. 
[2] Lezec H J, Dionne J A and Atwater H A 2007 Negative refraction at visible frequencies Science 316 430-432.

[3] Zhang X and Liu Z 2008 Superlenses to overcome the diffraction limit Nat. Mater. 7435 441.

[4] Shin D, Urzhumov Y, Jung Y, Kang G, Baek S, Choi M, Park H, Kim K, Smith D R 2013 Nat. Commun. 31213.

[5] Zhu Z, Yang X, Gu J, Jiang J, Yue W, Tian Z, Tonouchi M, Han J and Zhang W 2013 Broadband plasmon induced transparency in terahertz metamaterials Nanotechnology 24 214003

[6] Si G Y, Leong E S P, Pan W, Chum C C and Liu Y J 2014 Plasmon-induced transparency in coupled triangle-rod arrays Nanotechnology 26025201.

[7] Esfandyarpour M, Garnett E C, Cui Y, McGehee M D and Brongersma M L 2014 Metamaterial mirrors in optoelectronic devices Nature Nanotechnol. 9 542-547.

[8] Cao C, Zhang J, Wen X, Dodson S L, Dao N T, Wong L M, Wang S, Li S, Phan A T and Xiong Q 2013 Metamaterials-Based Label-Free Nanosensor for Conformation and Affinity Biosensing ACS Nano 7 7583-7591

[9] Chen Z, Guo B, Yang Y, Cheng C 2014 Metamaterials-based enhanced energy harvesting: A review Physica B: Condensed Matter 438 1-8

[10] McCrindle I J H, Grant J, Drysdale T D and Cumming D R S 2014 Multi-Spectral Materials: Hybridisation of Optical Plasmonic Filters and a Terahertz Metamaterial Absorber. Adv. Optical Mater 2 149-153

[11] Liu Y, Zhang X 2011 Metamaterials: a new frontier of science and technology Chem. Soc. Rev. 40 2494-2507. 
[12] Kanté B, de Lustrac A, Lourtioz J-M and Gadot F 2008 Engineering resonances in infrared metamaterials Opt. Express 16 6774-6784.

[13] Fan K, Strikwerda A C, Zhang X and Averitt R. D 2013 Three-dimensional broadband tunable terahertz metamaterials Phys. Rev. B 87 161104(R)

[14] Alici K B, Serebryannikov A E, Ozbay E 2011 Photonic magnetic metamaterial basics Phot. Nano. Fund. Appl. 9 15-21

[15] Yen T J, Padilla W J, Fang N, Vier D C, Smith D R, Pendry J B, Basov D. N and Zhang X 2004 Terahertz magnetic response from artificial materials Science 303 1494-1496 (2004).

[16] Withayachumnankul W and Abbott D 2009 Metamaterials in the Terahertz Regime. IEEE Photonic Journal 1 99-116

[17] Linden S, Enkrich C, Wegener M, Zhou J, Koschny T, Soukoulis C M 2004 Magnetic Response of Metamaterials at 100 Terahertz. Science, 306 1351-1353

[18] Okamoto T, Otsuka T, Sato S, Fukuta T and Haraguchi M 2012 Dependence of LC resonance wavelength on size of silver split-ring resonator fabricated by nanosphere lithography Opt. Express 20 24059-24067

[19] Chen H-T, O'hara J F, Azad A K, Taylor A J, Averitt R D, Shrekenhamer D B and Padilla W J 2008 Experimental demonstration of frequency-agile terahertz metamaterials. Nature Photon. 2 295-298.

[20] Aydin K, Pryce I M and Atwater H A 2010 Symmetry breaking and strong coupling in planar optical metamaterials Opt. Express 18 13407-13417.

[21] Busch K, Freymann G von, Linden S, Mingaleev S F, Tkeshelashvili L and Wegener M 2007 Periodic nanostructures for photonics Phys. Rep. 444 101-202. 
[22] Zhou J, Koschny T, Kafesaki M and Soukoulis C M 2008 Size dependence and convergence of the retrieval parameters of metamaterials. Phot. Nano. Fund. Appl. 6 96-101 [23] Larsen K, Austin D, Sandall I C, Davies D G, Revin D G, Cockburn J W, Adawi A M, Airey R J, Fry P W, Hopkinson M and Wilson L R 2012 Electrical modulation of the optical properties of mid-infrared metamaterials Appl. Phys. Lett. 101251109

[24] Gregory S. A, Stenning G B G, Bowden G J, Zheludev N I and de Groot P A J 2014 Giant magnetic modulation of a planar, hybrid metamolecule resonance. New J. Phys. 16 063002

[25] Seren H R, Keiser G R, Cao L, Zhang J, Strikwerda A C, Fan K, Metcalfe G D, Wraback M, Zhang X, Averitt R D 2014 Optically modulated multiband terahertz Perfect absorber Adv. Optical Mater 2 1221-1226

[26] Dicken M J, Aydin K, Pryce I M, Sweatlock L A, Boyd E M, Walavalkar S, Ma J, and Atwater H A 2009 Frequency tunable near-infrared metamaterials based on $\mathrm{VO}_{2}$ phase transition Opt. Express 17 18330-18339

[27] Gu J, Singh R, Liu X, Zhang X, Ma Y, Zhang S, Maier S A, Tian Z, Azad A K., Chen H-T, Taylo A J, Han J and Zhang W 2012 Active control of electromagnetically induced transparency analogue in terahertz metamaterials. Nat. Commun. 31151

[28] Lin P T, Singh V, Hu J, Richardson K, Musgraves J D, Luzinov I, Hensley J, Kimerling L C and Agarwal A 2013 Chip-scale Mid-Infrared chemical sensors using airclad pedestal silicon waveguides Lab Chip 13 2161-2166

[29] Andrea Centrone 2015 Infrared Imaging and Spectroscopy Beyond the Diffraction Limit Annu. Rev. Anal. Chem. 8 101-26 
[30] Kahn J M, and Barry J R 1997 Wireless Infrared Communications. Proc. IEEE 85 265298

[31] Grigorescu A E and Hagen CW 2009 Resists for sub-20-nm electron beam lithography with a focus on HSQ: state of the art Nanotechnology 20292001

[32] Yue W, Wang Z, Yang Y, Chen L, Syed A, Wong K and Wang X 2012 Electron-beam lithography of gold nanostructures for surface-enhanced Raman scattering J. Micromech. Microeng. 22125007

[33] Website: https://www.cst.com/Products/CSTmws/FIT

[34] Dayal G, Ramakrishna S A 2012 Design of highly absorbing metamaterials for infrared frequencies Opt. Express 20 17503-17508

[35] Kanté Br, Lourtioz J-Ml and Lustrac A de 2009 Infrared metafilms on a dielectric substrate. Phys. Rev. B 80205120

[36] Ordal M A, Long L L, Bell R J, Bell S E, Bell R R, Alexander R W, Jr. and Ward C A 1983 Optical properties of the metals $\mathrm{Al}, \mathrm{Co}, \mathrm{Cu}, \mathrm{Au}, \mathrm{Fe}, \mathrm{Pb}, \mathrm{Ni}, \mathrm{Pd}, \mathrm{Pt}, \mathrm{Ag}, \mathrm{Ti}$, and $\mathrm{W}$ in the infrared and far infrared Appl. Opt. 22 1099-1119

[37] Skauli T, Kuo P S, Vodopyanov K L, Pinguet T J, Levi O, Eyres L A, Harris J S, Fejer M M, Gerard B, Becouarn L and Lallier E 2003 Improved dispersion relations for GaAs and applications to nonlinear optics J. Appl. Phys. 94 6447-6455

[38] Cai W and Shalaev V 2009 Optical Metamaterials: Fundamentals and Applications (New York, Springer) page 94.

[39] Zhang X, Li Q, Chao W, Yue W, Gu J, Tian Z, Han J and Zhang W 2011 Equivalent circuit analysis of terahertz metamaterial filters Chin. Opt. Lett. 9110012 